\title{
Intrapelvic Lumbosacral Fracture Dislocation in a Neurologically Intact Patient: A Case Report
}

\author{
Ahmad Safaie Yazdi ${ }^{1}$; Farzad Omidi-Kashani ${ }^{2,}$; Aslan Baradaran $^{2}$ \\ ${ }^{1}$ Department of Neurosurgery, Farabi Hospital, Islamic Azad University, Mashhad, IR Iran \\ ${ }^{2}$ Orthopedic Research Center, Orthopedic Department, Imam Reza Hospital, Mashhad University of Medical Sciences, Mashhad, IR Iran \\ *Corresponding author. Farzad Omidi-Kashani, Orthopedic Research Center, Orthopedic Department, Imam Reza Hospital, Mashhad University of Medical Sciences, Mashhad, IR Iran. \\ P.O.Box: 9137913316. Tel: +98-5137646500, Fax: +98-5138595023, E-mail: omidif@mums.ac.ir
}

Received: November 18, 2014; Revised: February 7, 2015; Accepted: April 18, 2015

\begin{abstract}
Introduction: Lumbosacral fracture dislocation is a rare entity mainly occurred in high-energy trauma accidents. In this unstable injury, anatomical separation of the spinal column from pelvis is usually associated with severe neurological deficits.

Case Presentation: We described a 16-year-old girl with extremely severe axial trauma to the lumbosacral spine who presented with fracture dislocation of the lumbosacral spine and its intrusion to the pelvic space. Despite violent lumbosacral joint dissociation on imaging studies, the patient was neurologically intact. She was treated with spinopelvic fusion and instrumentation.

Conclusions: Although spinopelvic fracture dislocation injuries are severe high-energy entities, in cases with traumatic spondylolytic spondylolisthesis due to widening of the vertebral canal, neurologic deficit may not be seen at all.

Keywords: Lumbosacral Region; Fractures; Dislocation; Injuries
\end{abstract}

\section{Introduction}

Lumbosacral fracture dislocation is a rare entity mainly occurred in high-energy trauma accidents (1-4). Intimate and strong osteoligamentous elements exist in this especial area of spine protecting neural elements that are responsible for the innervations of lower extremities, bowel, bladder and sexual organs (5). Roy-Camille et al. was the first to describe this injury as a "suicide jumper's fracture" (1). In these unstable injuries, anatomical separation of the spinal column from pelvis is usually associated with severe neurological deficits (6). Here, we described a 16-year-old girl with extremely severe axial trauma to the lumbosacral spine who presented with fracture dislocation of the lumbosacral spine and its intrusion to the pelvic space. Despite violent lumbosacral joint dissociation on imaging studies, our patient was neurologically intact.

\section{Case Presentation}

A 16-year-old girl presented to our emergency department with right heel and low back pain due to a fall from the fifth floor of a building. She had no history of unconsciousness or headache and vital signs were stable and satisfactory. Her past medical history was unremarkable. On physical examination, head and neck, thorax, upper extremities, and abdomen were all normal. Neurological examination was unremarkable for sensory loss in the lower extremities or saddle area. Sphincter function and mo- tor power in big toe extensors and other lower extremity muscles were normal and deep tendon reflexes remained intact. She had no intention of committing suicide.

Imaging scans revealed a severe left calcaneal fracture (a bag of bones) and an amazing intrapelvic intrusion of lumbar spine (Figure 1).

Vertebral body of the fourth and fifth lumbar vertebrae in associated with the first sacral segment protruded anteriorly while posterior bony elements remained in their relatively normal places (traumatic spondylolisthesis). The calcaneal fracture was treated conservatively, but spinopelvic disruption underwent surgery.

\subsection{Surgical Technique}

Paravertebral muscles were retracted bilaterally and loose laminar fragments resected for avoidance of later neural compression. Then pedicular screws were inserted in L4, L5 and iliac crest bilaterally under the biplane fluoroscopic control. Upper and lower screws were assembled by two longitudinal lordotic rods. Longitudinal traction was applied and then, the screws were tightened. Bone decortication was carried out and a mixture of allograft (chips cortico-cancellous allograft, tissue regeneration corporation; TRC, Kish, Iran) and autograft (derived from local bone) was placed on decorticated areas. Soft tissues were repaired in anatomical layers on a suction drainage. 

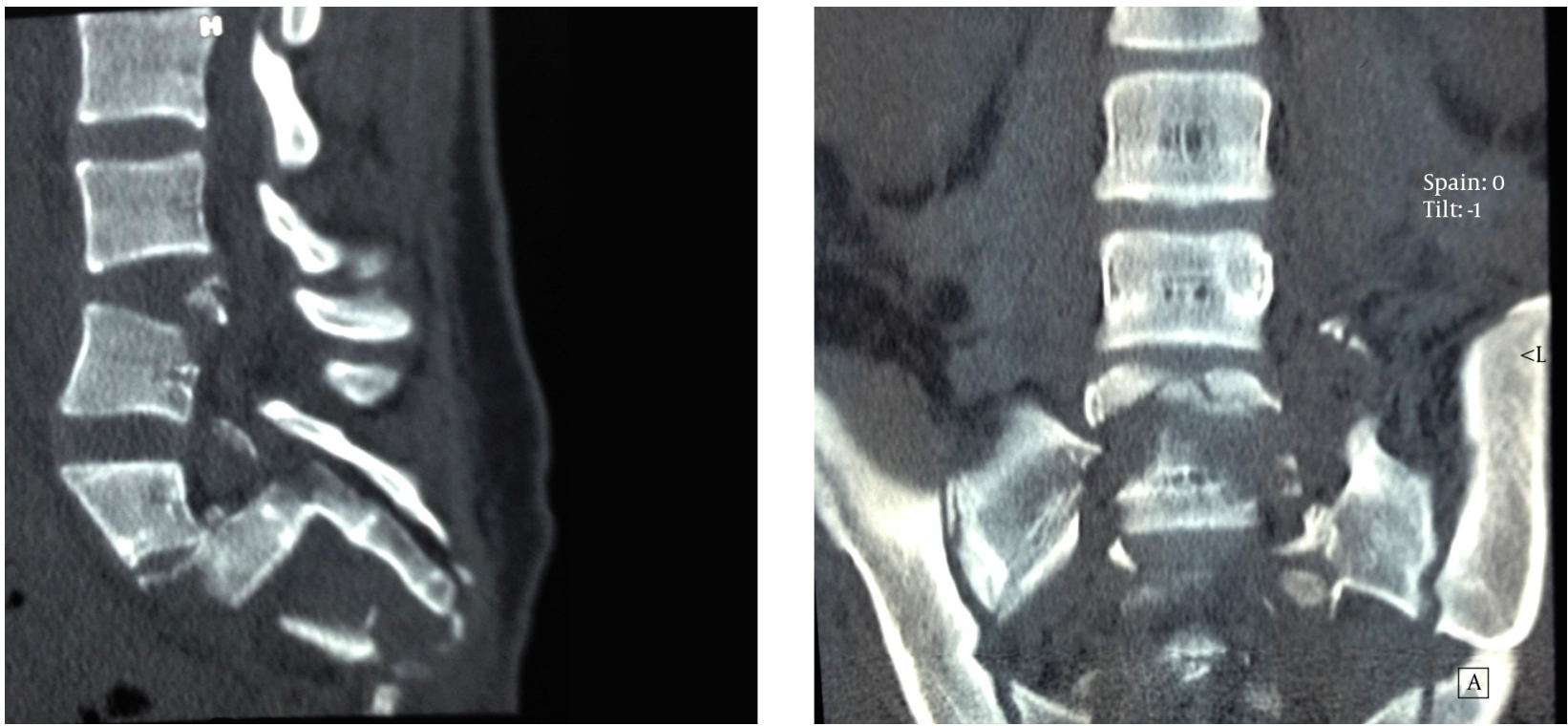

Figure 1. Sagittal and Coronal Computed Tomography Images Revealed Severe Intrapelvic Lumbosacral Displacement and Comminution

\subsection{Postoperative Course}

The patient was discharged three days after the operation with a rigid lumbosacral orthosis for more assurance. The brace was removed three months later. At the last follow-up visit 31 months later, the patient was pain free and able to walk easily without any significant complains; however, mild degrees of ankle pain due to the previous trauma remained, although mild degrees of ankle pain due to the previous trauma remained. Radiologic imaging revealed a right sided broken rod due to the underlying pseudoarthrosis (Figure 2).

On physical examination, neurological status was remained completely intact and she could do her routine activities of daily living without any problems.

\section{Discussion}

Vertebral fracture dislocation usually is the most violent type of spinal injuries that commonly associated with spinal instability and neurological deficit (7). In this type of injury, due to severe osteoligamentous disruption, stability of the spinal column is lost and probability of early and late neurological injury aggravated. In the case we reported, in spite of severe vertebral fracture and displacement, neurologic injury was not present at all. In a similar case reported by Acikbas and Gurkanlar, a posttraumatic C7-T1 spondyloptosis was happened in a patient without any neurologic deficit (8). They suggested the reason for this discrepancy between the pattern of injury and clinical finding is due to separation of anterior and posterior vertebral elements leaving the vertebral canal even broader. We also agree with these authors that the similar mechanism has induced severe osteoligamentous injury while the patient remained neurologically intact.
Figure 2. Lateral and Anteroposterior Views of Lumbosacral Spine 31 Months After Surgery
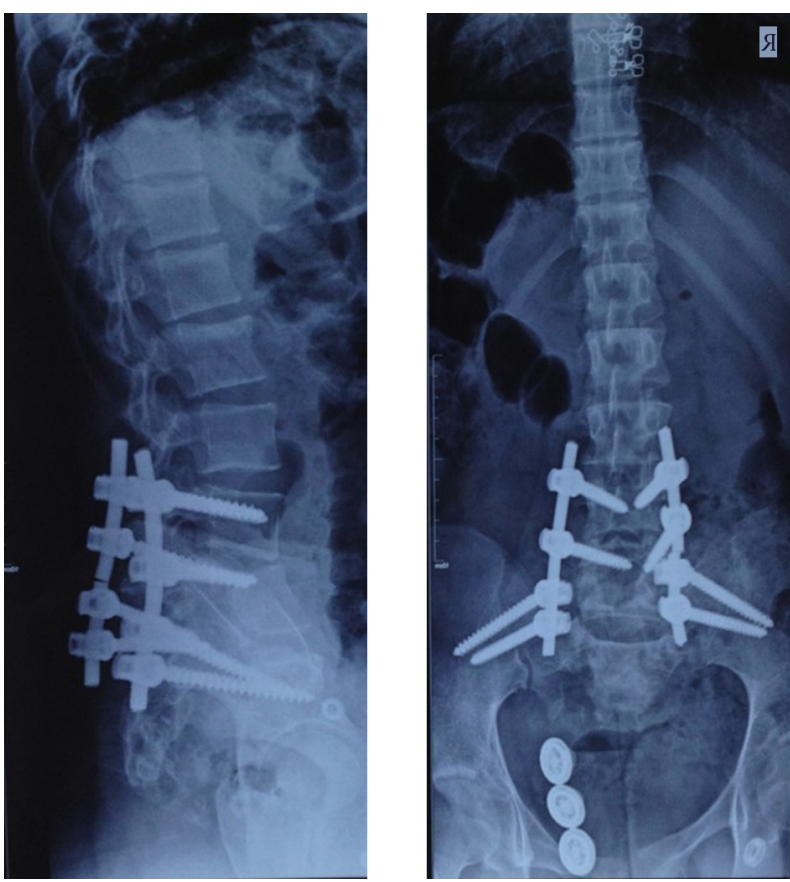

Failure of the right sided rod represented the underlying pseudoarthrosis, but the patient was asymptomatic clinically. 
As a significant amount of energy needed to produce these types of injury, stability of the spine is usually lost and surgical intervention became necessary. Herrera et al.in a case report described single level transforaminal interbody fusion for this type of injury (9). In the case they had reported, a unilateral L5 neurologic deficit was happened and the patient treated with open reduction, transforaminal lumbar interbody, and L5-S1 posterolateral instrumented fusion. They reported excellent result and recommended this type of surgery for comparable cases. On the contrary, the severity of osteoligamentous injury in our case was too significant to be treated by single level instrumentation and fusion. Sacrum with its great comminution was not able to support upper injured vertebrae and spinopelvic stability was inevitably needed. The occurrence of rod breakage in our case was probably due to our inability to remove superfluous soft tissue, decorticate bone efficiently, or apply sufficient bone graft, although the patient was fortunately asymptomatic and secondary operation was not necessary. In conclusion, although spinopelvic fracture dislocation injuries are severe high-energy entities, in cases with traumatic spondylolytic spondylolisthesis due to more widening of the vertebral canal, neurologic injury may not be seen at all.

\section{Authors' Contributions}

Ahmad Safaie Yazdi prepared the case, collected data and supervised the study. Farzad Omidi Kashani prepared the manuscript and performed the literature review. Aslan Baradaran involved in data collection and editing of the manuscript. All authors reviewed and confirmed the final manuscript.

\section{References}

1. Roy-Camille R, Saillant G, Gagna G, Mazel C. Transverse fracture of the upper sacrum. Suicidal jumper's fracture. Spine (Phila Pa 1976). 1985;10(9):838-45.

2. Helgeson MD, Lehman RAJ, Cooper P, Frisch M, Andersen RC, Bellabarba C. Retrospective review of lumbosacral dissociations in blast injuries. Spine (Phila Pa 1976). 2011;36(7):E469-75.

3. Schildhauer TA, Bellabarba C, Nork SE, Barei DP, Chip Routt ML, Chapman JR. Decompression and Lumbopelvic Fixation for Sacral Fracture-Dislocations With Spino-pelvic Dissociation. J Orthopa Trauma. 2006;20(7):447-57.

4. Wangtaphan W, Oo M, Paholpak P, Wang Z, Sakakibara T, Kasai Y. Traumatic lumbosacral spondyloptosis treated five months after injury occurrence: a case report. Spine (Phila Pa 1976). 2012;37(22):E1410-4.

5. Lehman RAJ, Kang DG, Bellabarba C. A new classification for complex lumbosacral injuries. Spine J. 2012;12(7):612-28.

6. Vaccaro AR, Kim DH, Brodke DS, Harris M, Chapman JR, Schildhauer T, et al. Diagnosis and management of sacral spine fractures. Instr Course Lect. 2004;53:375-85.

7. Wood KB, Li W, Lebl DR, Ploumis A. Management of thoracolumbar spine fractures. Spine J. 2014;14(1):145-64.

8. Acikbas C, Gurkanlar D. Post-traumatic C7-T1 Spondyloptosis in a patient without neurological deficit: a case report. Turk Neurosurg. 2010;20(2):257-60.

9. Herrera AJ, Berry CA, Rao RD. Single-level transforaminal interbody fusion for traumatic lumbosacral fracture-dislocation: a case report. Acta Orthop Belg. 2013;79(1):117-22. 